\title{
Hydrophobic mechanism and criterion of lotus-leaf-like micro-convex-concave surface
}

\author{
ZHU DingYi, QIAO Wei \& WANG LianDeng \\ Department of Materials Science and Engineering, Fuzhou University, Fuzhou 350108, China
}

Received July 15, 2010; accepted December 13, 2010; published online April 13, 2011

\begin{abstract}
According to the principle that the contact angle of liquid droplet always increases on a limited liquid-solid interface, it is suggested that the integration of many small-size limited liquid-solid interfaces results in the increase of the hydrophobicity of lotus-leaf-like micro-convex-concave surfaces. Mathematical equations of the stability of liquid-droplets on the surface of lotus-leaf-like structure were established. The relationship between the theoretical critical-radius of the void of micro-convex-concave surface and the nature of the solid and the liquid was drawn. The three conditions of realizing hydrophobicity were described. The result of computation has shown that when the radius of the void of micro-concave-convex surface is less than the theoretical critical-radius $r_{\mathrm{c}}$, the droplets may always be in a stable state on the solid surface with the contact angle greater than $90^{\circ}$. The minimum area of the liquid-solid interface and low surface energy of solids are important factors in realizing hydrophobicity. The effective work of adhesion $W_{a}^{\prime}$ was proposed as a criterion for measuring the hydrophobic ability of the solid surface.
\end{abstract}

limited liquid-solid interfaces, hydrophobicity, contact angle, critical radius, work of adhesion

Citation: Zhu D Y, Qiao W, Wang L D. Hydrophobic mechanism and criterion of lotus-leaf-like micro-convex-concave surface. Chinese Sci Bull, 2011, 56: 1623-1628, doi: 10.1007/s11434-011-4464-5

Water droplets fall on lotus leaf surface to form free rolling water globules. The exploration on hydrophobic mechanism of the lotus leaf surface has become a hot spot in the field of artificially preparing bionic hydrophobic and self-cleaning materials. In recent years, the research on new hydrophobic materials has continually achieved remarkable achievements in the fields of chemical engineering, daily use sanitary wares, clothing, fluid drag reduction, mechanical anti-corrosion and so on. After long-term observations, the researchers have found [1-3] that there are very complex multiple micron and nano-scale structures on the lotus leaf surface. It can be clearly seen under the scanning electron microscope that the lotus leaf surface has many small papillae, on the surface of each of which there are a lot of "hill" shaped convex top with the diameter of about $200 \mathrm{~nm}$. The sunken parts between the papillae and the "hills" are filled

*Corresponding author (email: zdy7081@163.com) with air. When rainwater lands on the leaf surface, it only constitutes partial point contact with the "hill" shaped convex top on the leaf surface. Researches have shown that, the micro-nano-structured morphology exists not only in the lotus leaf surface but also in the surface of other plants and skin of some animals [4]. However, the current research only clarifies the special constructs of the surface of hydrophobic materials, that is, the hydrophobic material should have a rough surface and low surface energy [5,6]. However, researches on the reason why the contact angle of rough surface is greater than that of smooth surface and the quantitative researches on the microstructure size are not sufficient. Wenzel [7] proposed that water droplets were completely wetted on the rough surface, whose apparent contact angle could be expressed as: $\cos \theta_{r}=r \cos \theta$, where $\mathrm{r}$ was the surface roughness. He considered that, for the hydrophobic surface, due to $\theta \geqslant 90^{\circ}$, increasing the surface roughness $r$ would cause the increase of the apparent contact angle $\theta_{r}$ of 
liquid droplets, while for the hydrophilic surface, due to $\theta \leqslant 90^{\circ}$, increasing the surface roughness $r$ would cause the decrease of contact angle $\theta_{r}$ of liquid droplets. Cassie [8] believed that there existed two interfaces when water droplets were on the rough surface, namely, the contact interface between the water droplets and the solid and the interface between the water droplets and the air formed because the water droplets were not able to enter microscopic holes due to the capillary phenomenon. The contact angle of water droplets on the rough surface was expressed as: $\cos \theta_{r}=$ $-1+\Phi_{s}(\cos \theta+1)$, where $\Phi_{s}$ was the proportion of the solid-liquid interface. Cassie' model showed that, the smaller the $\Phi_{s}$ value was, the larger the apparent contact angle $\theta_{\mathrm{r}}$ was. However, it is not clear what conditions have led to hydrophilic or hydrophobic state and the transformation thereof. And there is no specific quantitative concept about the value of the definite solid surface roughness. Aiming at resolving the problems existed in the current research, the study explored the hydrophobic mechanism of lotus-leaf-like surface on the basis of the researches on the wetting system $[9,10]$ of limited liquid-solid interface.

\section{The principle that the apparent contact angle of liquid always increases on a limited liquid- solid interface}

The study established the wettability characterization system of a limited liquid-solid interface in the literature $[9,10]$, as shown in Figure 1. The liquid is located on a finite cylindrical solid surface, and the angle between Surface A and Surface B of the cylindrical solid is $90^{\circ}$. It is concluded from the calculations and experiments that under the equilibrium state $\left(\theta_{2} \rightarrow \theta_{\max }\right.$, the maximum contact angle of the liquid on a limited liquid-solid interface without loss), the numerical range of the contact angle of any liquid phase on the limited solid phase surface is $90^{\circ} \leqslant \theta_{2} \leqslant 180^{\circ}$. Compared with the wetting system of the infinite solid surface (Young's wetting system) shown in Figure 2, the apparent contact angle $\theta$ significantly increases, and the mathematical relationship [9] between $\theta_{2}$ and $\theta_{1}$ is

$$
\cos \theta_{1}=\sin \theta_{2}+\cos \theta_{2} .
$$

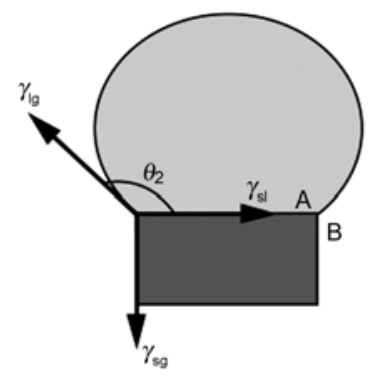

Figure 1 The wetting state of liquid on the limited liquid-solid interface.

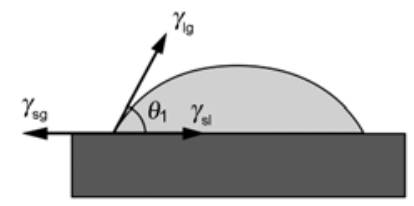

Figure 2 The wetting state of liquid on the infinite solid interface.

Figure 3 shows the functional relationship curve between $\theta_{2}$ and $\theta_{1}$. Eq. (1) shows that in the limited liquid-solid interface, when $\theta_{2} \rightarrow \theta_{\max }$, the apparent contact angle of any liquid will be greater than $90^{\circ}$. This characterization and calculation method is verified in the wettability tests of four high surface energy solids [11] and six high polymers [12] to obtain highly consistent calculation results.

Neinhuis and Feng et al. [1-3] made a research on the microcosmic morphology of the lotus leaf surface by the scanning electron microscope, and found that a large number of papillae with average diameter of 5-9 $\mu \mathrm{m}$ were orderly distributed on the lotus leaf surface. The sunken void was arranged between the papillae, the surface of each of which was distributed with villi with a diameter of hundreds of $\mathrm{nm}$, between which there were even smaller voids. The authors of this study believe that such a micro-concaveconvex surface is actually a surface integrated by a large number of limited liquid-solid interfaces. According to eq. (1), the contact angle of liquid droplets on the surface of this structure would increase, which is the reason why the apparent contact angle of liquid droplets on the micro-concaveconvex solid surface increases and also the first condition achieving the hydrophobicity. These papillary, fiber strip shaped $[4,13]$ and network-like $[14,15]$ limited liquid-solid interface structures have been verified in a large number of hydrophobic materials. Recently, the measurement results of the contact angle of the hydrophobic surface of chemically etched H62 brass showed [16] that the maximum contact angle obtained on the ladder-like rough surface was $131.8^{\circ}$, which was very close to the theoretical calculation value of $129.8^{\circ}$ obtained by eq. (1), which indicated that, the theory of limited liquid-solid interface could well explain

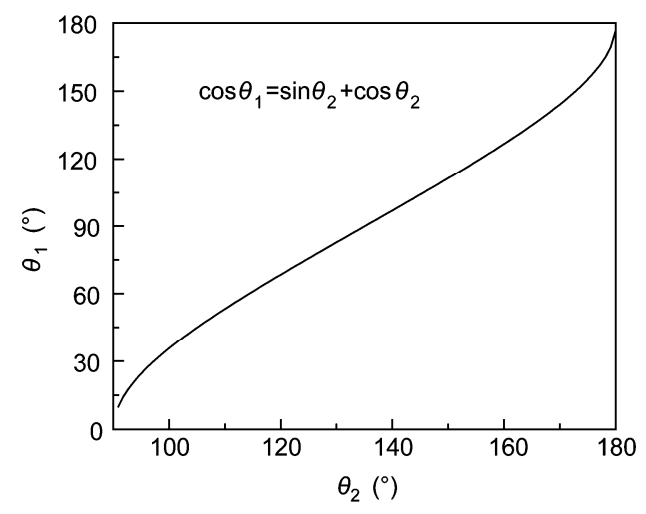

Figure 3 The functional relationship between $\theta_{2}$ and $\theta_{1}$. 
the reason why rough surface contact angle increased.

\section{The theoretical critical-radius of the void}

According to the micro-morphology of the lotus leaf surface, Patankar [17] further characterized the hydrophobic surface into micro-nanometer secondary columnar structure model, whose characteristics were shown in Figure 4. This kind of surface was actually a surface integrated by more micro-sized limited liquid-solid interfaces.

However, one of the conditions for the hydrophobic state of liquid droplets on the solid surface is that they cannot infiltrate into a large number of capillary microscopic holes because of wetting. If they do, the apparent contact angle thereof will become smaller and the rolling resistance thereof will increase. Whether infiltration can be avoided or not is closely related with the radius of the microscopic hole.

Figure 5 is a schematic diagram of the hydrophobic state of liquid droplets made in this study on the surface of the lotus-leaf-like structure. In order to facilitate the analysis, one unit of such a structure was here extracted for calculation, as shown in Figure 6.

It is assumed that at the first level of this secondary structure, there is a wafer-shaped limited liquid-solid interface with the thickness of $\delta$, on the center of which a hole with the radius of $r$ is arranged, and a liquid droplet with the radius of $R$ in the tension equilibrium state covers the surface of the solid. The upper surface of the wafer is the

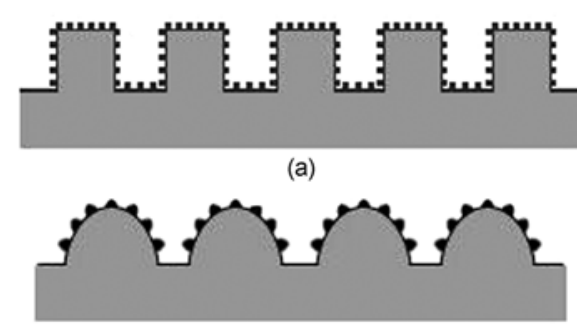

(b)

Figure 4 Double-column model of lotus-leaf-like surface. (a) Double-column model; (b) lotus-leaf-like model.

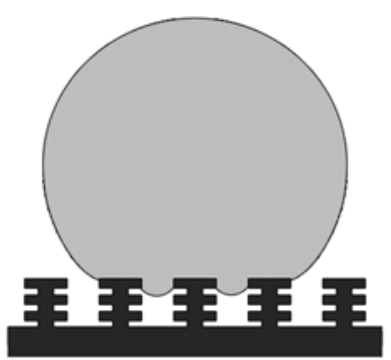

Figure 5 Schematic diagram of the hydrophobic state of droplets on the surface of the lotus-leaf-like structure.

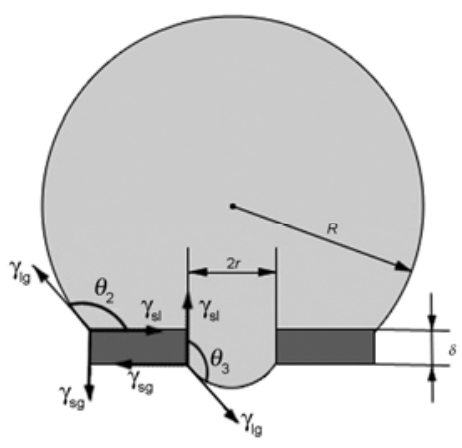

Figure 6 The surface tension distribution of a unit.

primary surface of the microstructure, while the side surface (first layer) of the central hole is the secondary surface. According to the balance of three-phase tension, the liquid would not stagnate in any part of the inner hole to achieve stability under the condition that the hole is a through-hole, that is, the liquid would pass through the inner hole to protrude downward which is supported by the upward tension component on the surface of the segment (anti-gravity direction). For the definite solid and liquid, under the influence of the 3 -phase $\left(\gamma_{\mathrm{sg}}, \gamma_{\mathrm{gg}}\right.$ and $\left.\gamma_{\mathrm{sl}}\right)$ surface (interface) tension, $\theta_{2}$ and $\theta_{3}$ in equilibrium are both constant and comply with the following relationships $[9,10]$ :

$$
\begin{gathered}
\gamma_{\mathrm{sl}}=-\gamma_{\mathrm{lg}} \cos \theta_{2}, 90^{\circ} \leqslant \theta_{2} \leqslant 180^{\circ}, \\
\gamma_{\mathrm{sg}}=\gamma_{\mathrm{lg}} \sin \theta_{2}, 90^{\circ}<\theta_{2} \leqslant 180^{\circ} .
\end{gathered}
$$

It is concluded from eqs. (2) and (3) that when $\theta_{2}$ and $\theta_{3}$ are in equilibrium, that is, when they are the maximum values, $\theta_{2}=\theta_{3}=\theta_{\max }$, the contact angle in the following study is expressed as $\theta$ if without any special descriptions.

It is assumed that due to gravity, once the actual contact angle $\theta$ is greater than $\theta_{\max }$, the state is unstable and the liquids will flow out of the hole and then infiltrate into more capillary microscopic holes at the sub-layer.

It is concluded from Figure 6 that the total tension on the surface of the segment preventing the liquids from flowing out of the hole with the radius of $r$ is $2 \pi r \cos \theta \gamma_{\mathrm{lg}}$.

And the liquid gravity exerted on the surface of the segment can be separated into 3 parts for calculation: the weight of the liquids of the segment part $m_{1}$, the weight of the liquids in the inner hole $m_{2}$, and the effective weight of liquid droplets on the columnar liquid-solid interface $m_{3}$, where $\quad m_{1}=\frac{\pi}{3} \rho r^{3} \frac{(1+\sin \theta)^{2} \cdot(2-\sin \theta)}{\cos ^{3} \theta}, m_{2}=-\pi r^{2} \rho \delta, m_{3}$ $=-\pi r^{2} \rho R(1-\cos \theta)$, where $\gamma_{\lg }$ is the liquid surface tension, $\rho$ is the density of the liquid, and $\theta$ values are $90^{\circ} \leqslant \theta \leqslant 180^{\circ}$. Because $m_{1}$ is a constant negative value, $m_{2}$ and $m_{3}$ should be set as negative values so as to maintain the gravity of each part of the liquid in the same direction. When the liquid droplets are in a stable state, that is, when the liquid 
droplets are just not able to flow out of the hole:

$$
\begin{aligned}
& \frac{\pi}{3} \cdot \rho \cdot r_{c}^{3} \frac{(1+\sin \theta)^{2} \cdot(2-\sin \theta)}{\cos ^{3} \theta} \\
& -\pi r_{c}^{2} \cdot \rho[\delta+R(1-\cos \theta)]=2 \pi r_{c} \cdot \cos \theta \cdot \gamma_{\mathrm{lg}} .
\end{aligned}
$$

From resolving eq. (4), the following equation is obtained:

$$
\begin{aligned}
r_{\mathrm{c}}= & 3 \cdot \cos ^{3} \theta \cdot\{\rho \cdot[\delta+R(1-\cos \theta)] \\
& -\sqrt{\left.\rho^{2} \cdot[\delta+R(1-\cos \theta)]^{2}+\frac{8}{3} \cdot \rho \cdot \gamma_{\mathrm{lg}} \cdot \frac{(1+\sin \theta)^{2} \cdot(2-\sin \theta)}{\cos ^{2} \theta}\right\}} \\
& \times\left[2 \cdot \rho \cdot(1+\sin \theta)^{2} \cdot(2-\sin \theta)\right]^{-1},
\end{aligned}
$$

$r_{\mathrm{c}}$ is the biggest critical radius when liquid droplets are in the stable state, which is the second condition for achieving hydrophobicity.

Eq. (5) shows that when the liquid droplets are in the stable state, the critical radius $r_{\mathrm{c}}$ of the central hole is a function of the contact angle $\theta$, the radius $R$ of the liquid droplet, and the thickness $\delta$ of the hole, in which $\theta$ depends on the nature of the liquid and the solid. Their mathematical relationship is reflected in eqs. (2) and (3).

When a water droplet covers a limited liquid-solid surface with microscopic holes, it is assumed that the thickness of the secondary structure in Figure 6 is $\delta=0.001 \mathrm{~m}$, and Figure 7 is a relationship curve between the critical radius $r_{c}$ of the hole and the contact angle $\theta$ when the water droplet is in the stable state, which is obtained by calculating eq. (5). The curves 1, 2, 3 and 4 in Figure 7 respectively represent the situation when the radius $R$ of the water droplet is 0.003 $\mathrm{m}, 0.005 \mathrm{~m}, 0.007 \mathrm{~m}$, and $0.01 \mathrm{~m}$, in which the surface tension and the density of the water are respectively 0.0728 $\mathrm{N} / \mathrm{m}$ and $9.8 \times 10^{3} \mathrm{~N} / \mathrm{m}^{3}$.

The result of computation shows that with the contact angle $\theta$ of the water droplet on the solid surface becoming

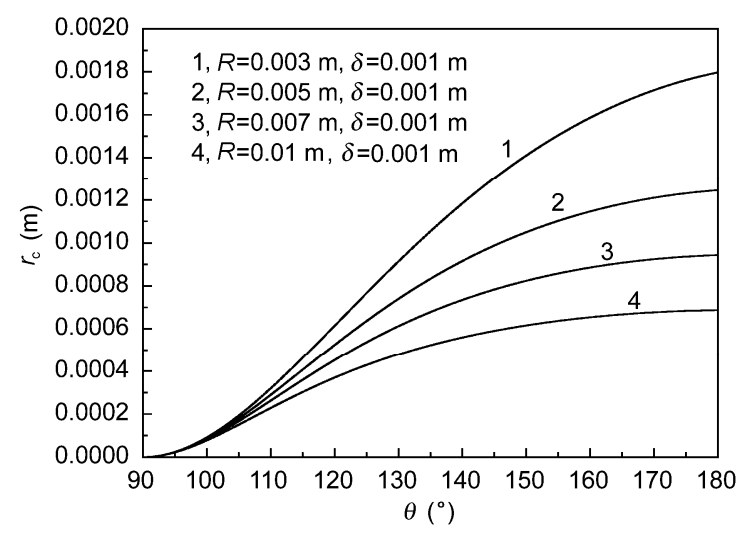

Figure 7 The relationship between critical radius $r_{\mathrm{c}}$ of the microscopic hole and the contact angle $\theta$ when the water droplet is in a stale state smaller, the critical pore diameter $r_{\mathrm{c}}$ when the water droplet is in a stable state becomes smaller; on the contrary, the more non-wetting the water droplet and solid have, the more bigger the critical pore diameter when the water droplet in a stable state. Due to the big contact angle $\theta$, the solid of low surface energy is favorable to realizing the hydrophobicity; secondly, the critical pore diameter $r_{\mathrm{c}}$ becomes smaller with the increase of the radius $R$ of the water droplet. Figure 7 shows that, in theory, the liquid droplet on any solid will stay in a stable state as long as the actual pore diameter of the solid is less than the theoretical one $r_{\mathrm{c}}$. This is a prerequisite for the free rolling of the liquid droplet.

The concave-convex surface of the actual hydrophobic materials is usually irregularly papillary. The included angle of the surface of each cylinder is not necessarily $90^{\circ}$ (right angle) as shown in the theoretical model of this study. When there is a cambered surface, due to the constant contact angle $\theta$, the apparent contact angle $\theta_{r}$ should be greater [16]: $\theta_{r}=\theta+\Delta \theta$, where newly increased $\Delta \theta$ is the angle between the cambered surface and the horizontal plane. Therefore, the contact angle of the surface of many practical hydrophobic materials is greater than $150^{\circ}$, which is called super hydrophobic state $[5,6]$. Eq. (5) shows that the influence of capillary thickness $\delta$ of the secondary surface on the size of the critical pore diameter is very small, while that of the thickness $\delta$ of the side surface of the inner hole on the rolling resistance of liquid droplets is bigger, indicating that the smaller the thickness $\delta$ is, the better the liquid droplets roll. The morphology of the lotus leaf shot by Feng et al. [3] showed that the average void between the lotus leaf papillae is about $15 \mu \mathrm{m}$, while the surface of each papilla is covered with many more subtle secondary microscopic hole structures, whose actual pore diameter was far less than the theoretical $r_{\mathrm{c}}$, and the thickness $\delta$ of the secondary surface is as small as the nano-scale, thus the lotus leaf surface is able to withstand much more water pressure and raindrops impact, but prevents the water droplets from infiltrating into microscopic holes to lose the rolling nature. If the holes on the solid surface are the blind ones, the gas in the microscopic holes can further prevent the infiltration of the liquids so that the hydrophobicity becomes better. Table 1 is the contact angles of the water droplet in a stable state with the radius of $5 \mathrm{~mm}$ and the corresponding radius of theoretical critical voids when it is on the solid surface of polytetrafluoroethylene, solid paraffin, polypropylene (PP), polystyrene (PS), and lime-soda organic glass, obtained from eq. (5), where the data of $\theta_{1}$ come from the literature [12], the numerical values of $\theta_{2}$ and $r_{\mathrm{c}}$ are respectively obtained by eqs. (1) and (5).

The result of computation shows that for the water droplet with the radius of $5 \mathrm{~mm}$, each solid has its own theoretical critical pore diameter. The critical radius $r_{\mathrm{c}}$ becomes larger with the increase of the contact angle $\theta$, so the low-energy 
Table 1 The contact angles of water in a stable state on several solid surfaces and the theoretical critical radius $(R=5 \mathrm{~mm})$ of the voids

\begin{tabular}{crcrrc}
\hline & \multicolumn{5}{c}{ Water } \\
\cline { 2 - 6 } & PTFE & Solid Paraffin & \multicolumn{1}{c}{ PP } & \multicolumn{1}{c}{ PS } & Synthetic glass \\
\hline$\theta_{1}\left({ }^{\circ}\right)$ & 106.62 & 100.20 & 94.49 & 91.87 & 80.06 \\
$\theta_{2}\left({ }^{\circ}\right)$ & 146.67 & 142.19 & 138.17 & 136.32 & 127.99 \\
$r_{\mathrm{c}}(\mathrm{mm})$ & 1.02 & 0.95 & 0.89 & 0.86 & 0.70 \\
\hline
\end{tabular}

modification of the solid surface is extremely important. If the average contact angle $\theta_{1}$ of the water droplet on the surface of solid paraffin is $100.2^{\circ}$, in the limited liquid-solid interface system, the contact angle $\theta_{2}$ obtained by calculating eq. (1) increases to $142.2^{\circ}$. Therefore, when the radius of the water droplet is $0.005 \mathrm{~m}$, the corresponding critical pore diameter $r_{\mathrm{c}}$ is $0.00095 \mathrm{~m}(0.95 \mathrm{~mm})$, indicating that as long as the radius of the microscopic hole is less than 0.95 $\mathrm{mm}$, the liquid droplet can maintain a stable state on the wax-filmed surface with the secondary composite structure as shown in Figure 7. However, the calculated value of the above theoretical pore diameter is much greater than the known one of the actual hydrophobic materials, because the calculation model is built at the theoretical critical state free of interferences of other physical factors except the gravity (static state). Moreover, the surface of the pore canal was designed to be ideally smooth and the included angle of the surface of each cylinder was $90^{\circ}$, while the surface of the actual hydrophobic materials is moderately curved rather than the sharp pore canal boundary, and the environment is very complex and harsh.

\section{The effective work of adhesion}

The fact that the limited liquid-solid interface causes the apparent contact angle of the liquid droplets to increase is only one of the conditions for realizing hydrophobicity. For small liquid droplets, a big apparent contact angle indicates a small actual liquid-solid contact area, while for the liquid of a large volume, due to the gravity, the actual liquid-solid contact area is very large and the flow resistance of the liquids increases. Therefore, the third condition for realizing hydrophobicity is to lower the surface energy of the solid and reduce the contact area of the liquid-solid interface as much as possible so as to reduce the flow resistance of liquid droplets on the solid surface. At present, this characteristic is often represented by the size of the rolling angle or the retardation angle [18], of which the two calculation methods are related to the values of the advance angle and the receding angle of the liquid droplets $[19,20]$. The authors of this study believe that this representation method did not reflect the internal causes affecting the hydrophobicity. The hydrophobicity is newly characterized quantitatively as follows: The difficulty in the rolling of liquid droplets on the solid surface depends essentially on the size of the work of adhesion $\mathrm{W}_{\mathrm{a}}$ between liquid-solid interfaces. In the infinite solid-phase plane system, the relationship is expressed by the well-known Young-Dupre Equation [9]:

$$
W_{a}=\gamma_{\lg }\left(1+\cos \theta_{1}\right), 0 \leqslant W_{a}<2 \gamma_{\lg }, 0<\theta_{1} \leqslant 180^{\circ} .
$$

The work of adhesion $W_{a}$ means the energy per unit needed for separating the liquid phase from the surface of the solid phase [9].

$$
W_{a}=\gamma_{\mathrm{sg}}+\gamma_{\mathrm{lg}}-\gamma_{\mathrm{sl}} .
$$

It can be seen that the smaller the work of adhesion is, the easier the liquid droplets can break away from the bondage of the solid surface to roll; the authors deduced in [9] the expression of the work of adhesion of the wetting system on the limited liquid-solid interface:

$$
W_{a}=\gamma_{\mathrm{lg}} \cdot\left(1+\sin \theta_{2}+\cos \theta_{2}\right), 90^{\circ}<\theta_{2} \leqslant 180^{\circ}, 0 \leqslant W_{a}<2 \gamma_{\mathrm{lg}} \text {. }
$$

The wetting systems characterized by eq. (8) and the Young-Dupre Equation are different, but the results are the same. When the nature of the liquid is determined, the work of adhesion mainly depends on the nature of the solid surface, while the solid with low surface energy can obtain a bigger contact angle.

Secondly, the smaller the actual liquid-solid interface is, the smaller the total work of adhesion is. There are a large number of holes on the hydrophobically good solid surface. Here, $\omega=A^{\prime} / A$ is called the effective liquid-solid interface fraction, where $A^{\prime}$ is the area of the actual liquid-solid interface excluding the holes, $A$ is the area of the liquid-solid interface without holes, and $0 \leqslant \omega \leqslant 1$. Therefore, the actual work of adhesion of the hydrophobic solid surface is

$$
W_{a}^{\prime}=W_{a} \cdot \omega=\gamma_{\mathrm{lg}} \cdot \omega \cdot\left(1+\sin \theta_{2}+\cos \theta_{2}\right), 90^{\circ}<\theta_{2} \leqslant 180^{\circ} .
$$

$W_{a}^{\prime}$, defined as the effective work of adhesion in this study, decreases with the decrease of $\omega$. Therefore, the hydrophobic ability of the solid surface can be measured with the size of the effective work of adhesion $W_{a}^{\prime}$ as a criterion. It reflects the internal reasons of the difficult levels of rolling of the liquid droplets, and the contact angle $\theta$ and the effective liquid-solid interface fraction $\omega$ can be obtained through the experimental measurement. Eq. (9) shows that the key technology of preparing lotus-leaf-like surface materials is to build structures of microscopic holes on the surface and minimize the liquid-solid interface area in addition to low-energy modification of the solid surface layer. Refs. [1-3] shows that the area of the void on the primary structure surface of lotus is greater than that of the papilla, the surface of the papilla of its secondary structure still has a large number of more thinner microscopic holes, which form many small air cushions, and there are few liquid-solid interfaces supporting the liquid droplets, so the lotus leaf surface has excellent hydrophobicity. 


\section{Conclusions}

(1) The fact that the liquids cover the solid surface integrated by a large number of limited liquid-solid interfaces is the reason why the apparent contact angle increases, which is the first condition for achieving hydrophobicity.

(2) For the definite wetting system, that the size of the void of the micro-concave-convex surface is smaller than the theoretical critical pore diameter ensures the liquid droplets are in a stable state, thus realizing the second condition for hydrophobicity. The result of computation shows that when the radius of the void of the micro-concave-convex surface is smaller than the critical radius $r_{\mathrm{c}}$ (the liquid droplets are in a stable state), the hydrophobicity of the surface of any solid can be theoretically improved.

(3) The third condition for realizing hydrophobicity is to minimize the area of the actual liquid-solid interface and lower the surface energy of the solid. The size of the effective work of adhesion $W_{a}^{\prime}$ can act as a criterion for measuring the hydrophobic ability of the solid surface.

This work was supported by the National Natural Science Foundation of China (50471007).

1 Neinhuis C, Barthlott W. Characterization and distribution of waterrepellent, self-cleaning plant surfaces. Ann Bot, 1997, 79: 667-677

2 Barthlott W, Neinhuis C. Purity of the sacred lotus or escape from contamination in biological surfaces. Planta, 1997, 202: 1-8

3 Feng L, Li S H, Li Y S, et al. Super-hydrophobic surfaces: From natural to artificial. Adv Mater, 2002, 14: 1857-1860

4 Gao X F, Jiang L. Water-repellent legs of water striders. Nature, 2004, 432: $36-36$

5 Richard D, Clance C, Quéré D. Surface phenomena: Contact time of a bouncing drop. Nature, 2002, 417: 811

6 Singh S, Houston J, Vail F, et al. Super hydrophobicity: Drying transition of confined water. Nature, 2006, 442: 526

7 Wenzel R W. Resistance of solid surfaces to wetting by water. Ind Eng Chem, 1936, 28: 988-994

8 Cassie A B D, Baxter S. Wettability of porous surfaces. Trans Faraday Soc, 1944, 40: 546-551

9 Zhu D Y, Dai P Q, Luo X B, et al. Novel characterization of wetting properties and the calculation of liquid-solid interface tension (I) (in Chinese). Sci Tech Eng, 2007, 7: 3057-3062

10 Zhu D Y, Zhang Y C, Dai P Q, et al. Novel characterization of wetting properties and the calculation of liquid-solid interface tension (II) (in Chinese). Sci Tech Eng, 2007, 7: 3063-3068

11 Luo X B, Zhu D Y, Qiao W, et al. Wettability of high surface energy solid and its surface tension calculation (in Chinese). J Mater Sci Eng, 2008, 26: 932-936

12 Zhang Y C, Zhu D Y, Xu S N. Experiment of wettability of high polymer surface and calculation of surface tension (in Chinese). Sci Tech Eng, 2009, 9: 3595-3600

13 Lim J M, Yi G R, Moon J H, et al. Superhydrophobic films of electrospun fibers with multiple-scale surface morphology. Langmuir, 2007, 23: 7981-7989

14 Feng L, Zhang Z, Jiang L, et al. A super-hydrophobic and superoleophilic coating mesh film for the separation of oil and water. Angew Chem, 2004, 116: 2012-2014

15 Minglin M, Randal M H, Joseph L L, et al. Electrospun poly (Styrene-block-dimethylsiloxane) block copolymer fibers exhibiting superhydrophobicity. Langmuir, 2005, 21: 5549-5554

16 Qiao W, Zhu D Y, Wen H Y, et al. Fabrication of a hydrophobic surface on H62 brass and study on hydrophobic mechanics (in Chinese). Sci Tech Eng, 2009, 9: 7319-7324

17 Patankar N A. Mimicking the lotus effect: Influence of double roughness structures and slender pillars. Langmuir, 2004, 20: 8209-8213

18 Chen C H, Cai Q J, Tsai C, et al. Dropwise condensation on superhydrophobic surfaces with two tier roughness. Appl Phys Lett, 2007, 90: 173108

19 Furmidge C G L. Studies at phase interfaces.I. The sliding of liquid drops on solid surfaces and a theory for spray retention. J Colloid Sci, 1962, 17: 309-324

20 Aussillous P, Quéré D. Liquid marbles. Nature, 2001, 411: 924-927

Open Access This article is distributed under the terms of the Creative Commons Attribution License which permits any use, distribution, and reproduction in any medium, provided the original author(s) and source are credited. 